\title{
Les dilemmes de l'insertion par l'activité économique : à la recherche du meilleur compromis
}

Work integration social enterprises facing their dilemmas : Pursuing the best compromise

Das Dilemma der Eingliederung durch Berufstätigkeit : auf der Suche nach dem besten Kompromiss

Los dilemas de la inserción por la actividad económica : en busca del mejor compromiso

\section{Philippe Semenowicz}

\section{OpenEdition}

Journals

Édition électronique

URL : http://journals.openedition.org/formationemploi/5327

DOI : 10.4000/formationemploi.5327

ISSN : 2107-0946

Éditeur

La Documentation française

Édition imprimée

Date de publication : 15 avril 2018

Pagination : 175-192

ISSN : 0759-6340

Référence électronique

Philippe Semenowicz, « Les dilemmes de l'insertion par l'activité économique : à la recherche du meilleur compromis », Formation emploi [En ligne], 141 | Janvier-Mars 2018, mis en ligne le 15 avril 2020, consulté le 30 octobre 2020. URL : http://journals.openedition.org/formationemploi/5327 ; DOI : https://doi.org/10.4000/formationemploi.5327 


\section{Les dilemmes de l'insertion par l'activité économique : à la recherche du meilleur compromis}

PhilipPe Semenowicz Agrégé de sciences sociales, docteur en sciences économiques, université Paris-Est Marnela-Vallée, ERUDITE (Equipe de recherche sur l'utilisation des données individuelles en lien avec la théorie économique ) - UPEMLV, F, 77454 Marne-la-Vallée

Résumé

Les dilemmes de l'insertion par l'activité économique : à la recherche du meilleur compromis

Les structures d'insertion par l'activité économique (SIAE) proposent à des personnes rencontrant des difficultés sociales ou professionnelles particulières une mise en situation de travail et une prise en charge visant la résolution de leurs diverses problématiques. À partir du cadre théorique de l'économie des conventions, nous analysons les compromis par lesquels les SIAE gèrent les tensions émanant de la poursuite d'objectifs multiples, comme, notamment, la viabilité financière de la structure, le bon accomplissement de la production, le retour à l'emploi des personnes en insertion. D'un point de vue empirique, nous nous appuyons sur deux monographies.

Mots clés : accompagnement professionnel, formation professionnelle continue

Abstract

Work integration social enterprises facing their dilemmas: Pursuing the best compromise

Work integration social enterprises (WISE) aim to allow disadvantaged workers to benefit from contracts of employment, in order to facilitate their social and professional inclusion. They have to handle the tensions occurred by the pursuing of multiple goals: reaching financial viability and efficiency, getting disadvantaged workers employable... We build a framework inspired from the "economics of conventions" school to investigate which compromises are needed. Our paper is based on two casestudies of French WISEs.

Keywords: job entry via a rehiring firm, economy of conventions, rehiring firm, temporary employment, coaching, continuing vocational training

Journal of Economic Literature; J 24; M 51 ; M 54

Traduction : Auteur. 


\section{Introduction}

L'insertion par l'activité économique (IAE) est née, au milieu des années soixante-dix, de diverses initiatives développées par la société civile afin de lutter contre l'exclusion professionnelle d'une partie de la population active. Son principe est de combiner, au sein de structures d'insertion par l'activité économique (SIAE), une production de biens ou de services avec une mission d'intérêt général, soit l'insertion de personnes rencontrant des difficultés sociales ou professionnelles particulières.

Accueillis pour une durée limitée à vingt-quatre mois maximum, les publics y sont à la fois mis en situation de travail, suivis pour la résolution de leurs diverses problématiques et aidés dans leur recherche d'un emploi de droit commun (cf. Encadré 1). Par opposition aux entreprises " classiques", dont l'objectif principal est la recherche du profit, les SIAE sont généralement considérées comme des entreprises sociales. Leur raison d'être réside dans l'accomplissement de leur mission (Bucolo \& al., 2012).

L'IAE constitue un champ hétérogène du point de vue de ses principes et de ses pratiques. Ses pionniers, majoritairement issus du travail social, valorisaient son apport en termes de socialisation, l'activité proposée permettant en effet de créer du lien et de conférer aux personnes en insertion une identité (Eme, 1994). Cette conception a depuis été supplantée par celle du "sas vers l'emploi ", au fur et à mesure que l'IAE s'est institutionnalisée (Autès, 1999). Les pouvoirs publics l'ont en effet progressivement reconnue, soutenue et encadrée pour en faire finalement un volet de la politique de l'emploi ${ }^{1}$. En a découlé une évaluation de l'efficacité des SIAE à l'aune de leurs taux d'insertion professionnelle à la sortie.

Cette focalisation sur la fonction de sas amplifie, au sein de l'IAE, les tensions émanant de la poursuite d'objectifs multiples, notamment, la viabilité financière de la structure, le bon accomplissement de la production, le retour à l'emploi des personnes en insertion... Comment en effet combiner différentes logiques potentiellement contradictoires?

Pour répondre à cette question, nous nous appuyons sur deux monographies de SIAE (cf. Encadré 2), analysées au prisme du cadre théorique de l'économie des conventions $(\mathrm{EC})^{2}$. Tout d'abord, nous envisageons les SIAE à partir du concept de " convention

\footnotetext{
1. Ce mouvement devrait se poursuivre suite au rapport "Donnons-nous les moyens de l'inclusion", remis à la ministre du Travail, le 16 janvier 2018, par Jean-Marc Borello. Il recommande un "pacte d'ambition " avec le secteur de l'IAE, qui vise une croissance annuelle de $20 \%$ du nombre de ses salariés jusqu'à la fin du quinquennat, mais moyennant "une hausse budgétaire plus modérée, grâce à une plus grande performance ".

2. L'EC est une école de pensée en sciences économiques, née dans les années quatre-vingt, centrée sur les modalités de coordination des acteurs. Elle mobilise le modèle des économies de la grandeur, développé par Boltanski et Thévenot (1991). À partir de la philosophie politique, ces auteurs ont distingué six « cités » (marchande, industrielle, domestique, civique, de l'opinion et inspirée) qui constituent autant de registres de justification pour les acteurs dans leurs prises de décision.
} 
constitutive d'entreprise ", proposé par Eymard-Duvernay (2004). Elles apparaissent alors comme des entreprises spécifiques, dont la convention constitutive peut être qualifiée de "civique ", au vu de l'accompagnement qui est assuré en leur sein. Puis, nous examinons comment la mission sociale se combine avec la dimension économique de la structure. La confrontation des différentes logiques aboutit, au sein des SIAE, à l'émergence de compromis entendus comme "un principe capable de rendre compatibles des jugements s'appuyant sur des objets relevant de mondes différents " (Boltanski et Thévenot, op. cit., p. 338). Ces compromis visent à surmonter les dilemmes auxquels les SIAE sont confrontées.

\section{Encadré 1. L'organisation de l'IAE (Insertion par l'activité économique)}

La loi d'orientation du 29 juillet 1998, relative à la lutte contre les exclusions, propose une définition officielle de l'IAE à partir de trois critères. Tout d'abord, I'IAE s'adresse à des personnes sans emploi rencontrant des difficultés sociales ou professionnelles particulières et qui, de ce fait, ne sont pas susceptibles d'être embauchées par les entreprises classiques (la conformité des publics est contrôlée par un agrément délivré par Pôle emploi). Ensuite, elle offre à ces publics des contrats de travail à part entière, et non des stages de formation professionnelle ou de simples occupations rémunérées par un pécule. Enfin, elle leur propose également des modalités spécifiques d'accueil et d'accompagnement visant à résoudre les difficultés auxquelles ils sont confrontés. Ces modalités mobilisent des outils variés, comme la définition d'un projet, un entretien de suivi social, un bilan professionnel...

Parallèlement, sont proposées des formations de diverses natures, dispensées en interne comme en externe, tels que les savoirs de base, l'informatique, l'hygiène et la sécurité, le permis de conduire, l'adaptation au poste de travail, des formations qualifiantes ou pré-qualifiantes... (Rémy, 2016).

L'IAE recouvre diverses structures officiellement reconnues par les pouvoirs publics, comme les ateliers et chantiers d'insertion ( $\mathrm{ACl}$ ), les associations intermédiaires (Al), les entreprises d'insertion (EI), les entreprises de travail temporaire d'insertion (ETTI). Fin 2016, on comptait $3736 \mathrm{SIAE}$ ayant fait travailler, au cours de l'année, 132200 personnes (soit 73900 emplois équivalentstemps plein). Celles-ci exerçaient principalement dans les services à la personne et à la collectivité (35\%), l'agriculture, la pêche et l'entretien des espaces naturels et des espaces verts (18\%), la construction et le bâtiment travaux publics (BTP) (12\%), le transport et la logistique (10\%) (Bellit, 2017).

Les SIAE font l'objet d'un conventionnement avec les pouvoirs publics, réformé par le décret du 21 février $2014\left(^{*}\right)$. Son principal changement est de généraliser à toutes les SIAE le principe d'une aide au poste d'insertion, alors que chacune bénéficiait précédemment de modalités de financement spécifiques. L'aide au poste d'insertion varie selon le type de structure : 10237 euros pour une El, 1331 euros pour une Al, 4351 euros pour une ETTI et 19655 euros pour un $\mathrm{ACl}$ (montants par an et par équivalent temps plein en insertion pour l'année 2017). Cette aide peut de plus être modulée (jusqu'à $10 \%$ à la hausse).

${ }^{*}$ ) : Décret $n^{\circ}$ 2014-197 du 21 février 2014 portant généralisation de l'aide au poste d'insertion et diverses mesures relatives à l'insertion par l'activité économique. 


\section{Encadré 2. Méthodologie}

Notre enquête de terrain s'est déroulée d'août 2012 à avril 2014. Nous avons réalisé deux monographies de SIAE (Structures d'insertion par l'activité économique) basées en Île-de-France : une El (entreprise d'insertion) et une ETTI (entreprise de travail temporaire d'insertion). Au moment de l'enquête, l'El se composait de trois antennes et employait 210 salariés en insertion, pour un chiffre d'affaires de près de 10 millions d'euros. L'ETTI comptait également trois antennes et salariait 160 intérimaires équivalents-temps plein, pour un chiffre d'affaires de 5,3 millions d'euros. Si nous n'avons couvert que la moitié des types de SIAE, l'échantillon retenu tient compte de la distinction couramment opérée, au sein de $\mathrm{I}^{\prime} \mid \mathrm{AE}$, entre les structures assurant une production en interne (El, $\mathrm{ACl}$ - ateliers et chantiers d'insertion) et celles effectuant des mises à disposition auprès d'utilisateurs ( $\mathrm{Al}$ - association intermédiaire, ETTI).

Les SIAE enquêtées ont été sélectionnées sur deux critères. D'une part, elles adhèrent pleinement au modèle du « sas vers l'emploi » et incarnent donc deux exemples emblématiques de la conception de l'IAE promue par les pouvoirs publics. D'autre part, elles sont spécialisées sur les services aux entreprises classiques qui constituent leurs seuls clients. De ce fait, les exigences en matière de prix et de qualité de la prestation s'y avèrent plus élevées que dans les Al ou les $\mathrm{ACl}$, dont les clients sont majoritairement des particuliers ou des collectivités locales. Ces deux caractéristiques font des structures étudiées un terrain privilégié pour observer comment les SIAE articulent leur mission sociale avec leur dimension économique.

Nous avons analysé la documentation interne de chaque structure et réalisé, au total, 37 entretiens semi-directifs, aussi bien avec les dirigeants, administrateurs et salariés permanents de ces SIAE qu'avec leurs interlocuteurs au sein des entreprises classiques et du service public de l'emploi. En amont de chacun de ces entretiens, nous avons élaboré un guide adapté à la fonction de la personne interviewée. Les thèmes abordés dans le cadre des entretiens concernaient les modalités d'accompagnement en SIAE et les relations avec les entreprises clientes. Les entretiens, d'une durée moyenne d'une heure, ont ensuite été intégralement transcrits, puis analysés à la fois individuellement et thématiquement. Étant donné que l'objet du texte est d'étudier le fonctionnement interne des SIAE, nous nous concentrons ici uniquement sur les 19 entretiens réalisés avec leurs salariés permanents.

\section{Les SIAE reposent sur une convention constitutive civique}

Afin de disposer d'un outil d'analyse des SIAE, nous présentons tout d'abord le concept de "convention constitutive d'entreprise". Nous montrons ensuite, à partir de l'analyse de l'accompagnement qui s'y déroule, que la spécificité des SIAE est de reposer sur une convention constitutive d'entreprise de nature civique.

\subsection{Le concept de convention constitutive d'entreprise}

L'économie des conventions considère l'entreprise comme un espace politique où différentes conceptions du bien commun sont en concurrence. Selon Eymard-Duvernay, 
il existe en effet une "pluralité des conventions constitutives d'entreprise" (2004, p. 75), chacune constituant "des ressources de coordination" (Ibid., p. 72). L'auteur distingue ainsi quatre conventions constitutives d'entreprise : industrielle, marchande, domestique et de réseau.

Les trois premières conventions constitutives découlent de principes supérieurs communs dégagés par Boltanski et Thévenot (op. cit.). La convention constitutive marchande repose sur le principe supérieur commun de la concurrence que se livrent plusieurs entreprises pour satisfaire des clients. La convention constitutive industrielle correspond au modèle de l'entreprise fordiste, dans laquelle l'efficacité constitue le principe supérieur commun. La convention constitutive domestique se caractérise quant à elle par une valorisation de la tradition, de la proximité et d'un savoir-faire que l'on retrouve principalement dans les activités artisanales. Enfin, la convention constitutive de réseau émane de la "cité par projets", ajoutée au modèle des économies de la grandeur par Boltanski \& Chiapello (1999). Elle repose sur la recherche d'une organisation flexible, dont l'aboutissement est le modèle de la "firme-réseau ", dans lequel une entreprise-pivot confie à une multitude de fournisseurs la réalisation de certains éléments du processus de production (Baudry, 1995). Cette organisation vise à se procurer, en externe, le temps des " projets " successifs de l'entreprise, les ressources nécessaires à leur bon accomplissement. Le principe supérieur commun sur lequel elle se fonde est de nature connexionniste.

Les SIAE se caractérisent par une cinquième convention constitutive que nous qualifions de " civique ». En effet, le principe supérieur commun dans la cité civique est la volonté générale, que Boltanski et Thévenot entendent comme la capacité d'une personne à se détourner de son intérêt personnel pour faire prévaloir l'intérêt général. Même s'il ne l'applique pas au fonctionnement des entreprises, Eymard-Duvernay en fournit divers exemples : "La cité civique évalue les personnes et les biens par leur contribution à la protection des plus faibles: les syndicalistes, les membres des associations de défense des consommateurs, des immigrés, etc. sont les "grands" de cette cité" (op. cit., p. 84).

Or, sur le marché du travail, les plus faibles sont les demandeurs d'emploi en situation d'exclusion professionnelle, auxquels les SIAE se proposent d'offrir une protection. L'IAE a en effet pour objectif de remédier à la sélectivité du marché du travail, comme en témoigne sa devise selon laquelle "nul n'est inemployable». L'examen des caractéristiques des publics accueillis dans les deux SIAE étudiées (cf. Tableau 1) confirme leur éloignement de l'emploi. Ils sont ainsi majoritairement de niveau VI et Vbis ${ }^{3}$, avec une ancienneté dans le chômage élevée, et relèvent des "publics prioritaires " 4 .

3. Les niveaux VI et Vbis correspondent aux sorties en cours de 1er cycle de l'enseignement secondaire (6ème à 3ème) ou abandons en cours de CAP ou BEP (certificat d'aptitude professionnelle et brevet d'études professionnelles) avant l'année terminale.

4. On peut noter que les publics accueillis en ETTI sont moins éloignés de l'emploi qu'en EI : la proportion 
Dans les SIAE, la finalité sociale de l'entreprise favorise donc l'adoption de la convention civique comme mode de coordination interne. Toutefois, si le concept de convention constitutive insiste sur le fait qu'un principe supérieur commun domine dans l'entreprise, cette dernière se trouve également confrontée à d'autres principes de coordination. Chaque entreprise se caractérise donc par un compromis spécifique entre différents principes supérieurs communs.

Il en va également ainsi pour les SIAE 5 . Tout d'abord, comme elle vend sa production sur un marché, une SIAE doit prendre en compte le principe de la concurrence. Elle ne peut s'affranchir des conditions de prix pratiqués, sous peine de ne pas avoir de clients. Ensuite, une SIAE ne peut négliger le principe de l'efficacité, car elle doit mener à bien l'opération de production de biens ou de services dans laquelle elle est engagée. Ceci implique d'obtenir un certain niveau de productivité de la part des salariés en insertion, afin que la production visée soit effectivement assurée. Enfin, une SIAE est confrontée au principe connexionniste car elle est exposée aux transformations ayant affecté les entreprises classiques clientes. Elle doit se fondre dans l'organisation en réseau qui constitue alors le cadre de son intervention.

La convention constitutive d'entreprise de nature civique s'incarne dans les SIAE par l'existence d'un accompagnement des personnes en insertion. Il vise à la construction d'un "parcours d'insertion", élaboré avec les publics. Comme le notent Defalvard et Cervera (2009), l'accompagnement en SIAE est doublement dual. Tout d'abord, il est à la fois un accompagnement social et un accompagnement professionnel. Ensuite, il est également un accompagnement dans l'emploi et un accompagnement vers l'emploi. Il s'agit simultanément de permettre l'occupation d'un poste de travail et de préparer le retour à un emploi de droit commun. Nous décrivons maintenant ces diverses facettes de l'accompagnement.

de jeunes, de chômeurs de très longue durée ou de publics prioritaires y est en effet plus faible.

5. Par rapport à la typologie établie par Eymard-Duvernay (op. cit.), nous ne prenons pas ici en compte le principe domestique, caractérisé par la tradition, la proximité et un savoir-faire artisanal. Les conditions d'entrée, le caractère temporaire du passage en insertion et la nature des activités assurées constituent en effet des freins au développement du principe domestique dans les SIAE. 
Tableau 1. Caractéristiques des publics accueillis

\begin{tabular}{|l|l|l|}
\cline { 2 - 3 } \multicolumn{1}{c|}{} & \multicolumn{1}{c|}{ EI } & \\
\hline Répartition par sexe & & $96 \%$ \\
\hline Hommes & $78 \%$ & $4 \%$ \\
\hline Femmes & $22 \%$ & $20 \%$ \\
\hline Répartition par âge & & $69 \%$ \\
\hline Moins de 26 ans & $25 \%$ & $11 \%$ \\
\hline 26 à 50 ans & $63 \%$ & \\
\hline Plus de 50 ans & $12 \%$ & $61 \%$ \\
\hline Ancienneté dans le chômage & & $39 \%$ \\
\hline Inférieure à 2 ans & $50 \%$ & \\
\hline Supérieure à 2 ans & $50 \%$ & $62 \%$ \\
\hline Niveau d'études & & $25 \%$ \\
\hline VI et Vbis & $63 \%$ & $13 \%$ \\
\hline V & $23 \%$ & \\
\hline Supérieur à V & $14 \%$ & $24 \%$ \\
\hline Publics prioritaires & & $28 \%$ \\
\hline Bénéficiaires du RSA socle & $38 \%$ & $1 \%$ \\
\hline $\begin{array}{l}\text { Résidant zone urbaine sensible ou contrat } \\
\text { urbain de cohésion sociale }\end{array}$ & $42 \%$ & \\
\hline Travailleurs handicapés & & \\
\hline
\end{tabular}

Sigles : ETTI : entreprises de travail temporaire d'insertion ; El : entreprise d'insertion. RSA : revenu de solidarité active.

Lecture : 39 \% des salariés en insertion de l'ETTI étaient à l'entrée chômeurs de très longue durée (ancienneté de chômage supérieure à 2 ans).

Source : bilans adressés à la Direction régionale des entreprises, de la concurrence, de la consommation, du travail et de l'emploi (DIRECCTE) par l'El (2011) et l'ETTI (2013).

\section{2. Élaborer un projet professionnel}

L'accompagnement en SIAE est largement assuré par les salariés permanents occupant les fonctions de chargés d'insertion. Leur première tâche est de favoriser «la levée des freins à l'emploi ». Il s'agit de résoudre l'ensemble des difficultés rencontrées par les personnes en insertion et qui entravent l'exercice d'une activité professionnelle, comme les difficultés de logement ou d'accès aux soins, les problèmes administratifs ou judiciaires, les addictions, le surendettement... Dans cet objectif, les chargés d'insertion travaillent en partenariat avec d'autres organismes spécialisés sur ces différentes problématiques, vers lesquels ils orientent si nécessaire leurs publics. L'accompagnement se poursuit par l'élaboration du projet professionnel. Pour cela, les chargés d'insertion s'efforcent d'élargir les possibilités de choix par la découverte de nouveaux métiers. Ils effectuent aussi un repérage des compétences détenues par les personnes en insertion, comme cela a déjà été relevé par d'autres travaux ayant étudié les méthodologies du secteur de l'insertion (Baron et al., 1994) : "On va essayer de puiser dans toutes les petites ressources de chacun, dans leurs compétences, dans ce quils aiment faire. Voilà, essayer de chercher ce qui pourrait leur correspondre. Aussi voir par rapport aux expériences qu'ils ont pu acquérir durant leur parcours. On va essayer de travailler 
sur tout ça. » (Chargée d'accompagnement social, ETTI, titre professionnel de conseiller en insertion, un an d'expérience dans la fonction)

Le projet professionnel a pu être décrit comme un détour inutile (Castra, 2003), dont la fonction réelle serait de persuader les demandeurs d'emploi de se plier aux conditions proposées sur le marché du travail (Mauger, 2001). Dans les SIAE enquêtées, la réalité s'avère plus nuancée.

Si les contraintes du marché du travail sont prises en considération, le projet professionnel ne peut être réduit à une simple adaptation des personnes en insertion. Au sein de l'EI, le projet professionnel élaboré est testé via un dispositif de droit commun, soit l'évaluation en milieu de travail (EMT), qui consiste en un stage en situation. Il s'agit ainsi de garantir des possibilités de choix. L'EMT a en effet autant pour but de tester des aptitudes que de permettre à la personne en insertion de valider son projet en toute connaissance de cause, et donc d'y renoncer le cas échéant : "Voilà, c'est en permanence mettre le salarié face aux choix et contraintes et l'amener à se dire "C'est ça qui me plaît, mais je suis prêt à accepter telle ou telle contrainte et à faire tel ou tel sacrifice ou tel et tel effort". Et c'est absolument essentiel, parce que c'est impossible pour une personne d'avancer dans un projet, de mettre en place un certain nombre d'efforts si elle ne les a pas décidés ellemême. " (Responsable accompagnement social, EI, diplôme d'État d'assistante sociale, dix ans d'expérience dans la fonction)

De même, l'ETTI utilise l'intérim pour construire et tester le projet professionnel. Dans le cadre de son élaboration, la diversité des missions proposées peut en effet constituer un outil : "Quand on a une personne qui sait faire plein de choses, mais qui ne sait pas forcément vers quoi se diriger, ça va être aussi à nous de travailler, d'essayer de trouver une solution justement par rapport à ses compétences, ce qu'elle aura pu faire durant tout son parcours. Et permettre aussi de lui faire tester peut-être une mission d'une semaine en plomberie, peut-être une mission en tant qu'aide-maçon. Voilà, essayer de lui faire toucher un petit peu à tout pour voir ce qui peut lui correspondre. " (Chargée d'accompagnement social, ETTI, titre professionnel de conseiller en insertion, un an d'expérience dans la fonction)

Une fois le projet professionnel validé, sa concrétisation passe par la recherche d'un emploi de droit commun. Celle-ci se déroule en parallèle de l'occupation du poste de travail en SIAE. Dans cette optique, les chargés d'insertion initient leurs publics aux techniques de recherche d'emploi, comme la rédaction de CV et de lettres de motivation, l'utilisation de l'outil informatique, la simulation d'entretiens de recrutement...

\subsection{Développer des savoir-être davantage que des savoir-faire}

Dans les deux SIAE, l'accompagnement comprend des formations qui sont de plusieurs types. Tout d'abord, il existe des formations visant la maîtrise de savoirs de base (cours de français, élaboration d'un budget familial...). Ensuite, en fonction de leur 
projet professionnel, les salariés en insertion peuvent également bénéficier de formations pré-qualifiantes et qualifiantes. Ces dernières visent l'acquisition de savoir-faire susceptibles d'être mobilisés à la fois dans les tâches réalisées au sein de la SIAE et à la sortie sur le marché du travail : "Des fois, on dit "on le forme", "on va lui faire passer des habilitations", "on va lui faire passer un CACES6". C'est aussi pour que la personne monte en compétences. " (Responsable antenne $\mathrm{n}^{\circ} 1$, ETTI, DUT - diplôme universitaire de technologie, Techniques de commercialisation, trois ans d'expérience dans la fonction).

Pour autant, ce sont moins les savoir-faire que les savoir-être qui sont mis en avant par les salariés permanents des SIAE. Selon eux, sur les activités peu ou pas qualifiées visées à la sortie, les entreprises rechercheraient avant tout une main-d'œuvre fiable qu'elles se chargeraient de former ensuite aux tâches nécessaires : "En fait, c’est quelque chose que j'entends très souvent: "Ne vous inquiétez pas au niveau compétences. Nous on les formera sur le terrain. Mais je veux des mecs motivés et qui se comportent correctement". Et c'est vraiment ce que nous ressortent les entreprises le plus souvent. "(Responsable emploi et relations entreprises, EI, diplômée d'une grande école de commerce, deux ans d'expérience dans la fonction)

Ceci rejoint le constat d'Ebersold (2001), selon lequel, dans l'IAE, primerait l'inculcation de savoir-être, comme le respect des horaires, l'assiduité, la conformité aux consignes données, la capacité à travailler en équipe... Concernant leur acquisition, l'EI se distingue de l'ETTI par le fait que la première assure une production en interne, alors que la seconde effectue de la mise à disposition. Cette différence a une forte implication en matière d'accompagnement dans l'emploi. En effet, dans l'EI, des salariés permanents appelés encadrants techniques supervisent le travail des personnes en insertion, alors que dans l'ETTI, celui-ci s'effectue sous le contrôle de leurs responsables au sein de l'entreprise utilisatrice. Dans les deux cas, la transmission des savoirêtre a néanmoins en commun d'avoir pour support des tâches peu ou pas qualifiées. Les savoir-être vont être acquis progressivement au cours des différentes activités proposées : "On peut les affecter à des endroits en fonction des qualités comportementales qu'on attend qu'ils développent. " (Responsable accompagnement social, EI, diplôme d'État d'assistante sociale, dix ans d'expérience dans la fonction)

L'accompagnement dans les deux SIAE étudiées vise donc à résoudre les difficultés périphériques des personnes en insertion, à faciliter l'élaboration d'un projet professionnel et à permettre le développement de leurs compétences. Il s'agit ainsi de préparer le retour à l'emploi de droit commun pour des personnes qui étaient à l'entrée en situation d'exclusion professionnelle. Au sein des SIAE, cet accompagnement est l'expression d'une convention constitutive d'entreprise de nature civique. Celle-ci s'avère néanmoins en tension avec la prise en compte d'autres principes supérieurs communs.

6. Certificat d'aptitude à la conduite en sécurité, exigé pour l'utilisation d'engins de manutention. 


\section{Les SIAE, des entreprises vouées à d'insatisfaisants compromis?}

Au sein de toute entreprise, les principes marchand et industriel s'avèrent en confrontation : "La pluralité traverse une même entreprise, en tension entre des conceptions contradictoires de ce qui est bon pour l'entreprise, telles qu'elles émanent, par exemple, des ingénieurs et des commerciaux" (Eymard-Duvernay, op. cit., p. 75).

Dans les SIAE, ce conflit est majoré par la nécessité d'articuler principes marchand et industriel, non seulement entre eux, mais également avec le principe civique. Ceci est à l'origine de deux dilemmes spécifiques à l'IAE7 . D'une part, accepter tout type de contrat permet de garantir la viabilité financière de la structure, mais au risque d'un faible développement des compétences pour les personnes en insertion. D’autre part, sélectionner les personnes à l'entrée préserve la productivité des SIAE, mais en écartant les publics qui en ont le plus besoin. Établir des partenariats dans la durée avec les entreprises classiques peut néanmoins permettre d'atténuer ces dilemmes.

\subsection{Accepter tout type de contrat ?}

Dans chacune des deux SIAE étudiées, environ $80 \%$ des ressources proviennent de la vente de biens ou de services, le reste étant issu des aides publiques ou du mécénat. Cette importance des ressources marchandes dans le financement explique que ces SIAE se sont dotées d'un service commercial à part entière. Il s'agit ainsi d'obtenir de nouveaux contrats de façon à garantir la mise au travail des personnes en insertion et la viabilité financière de la structure. Si l'objectif est idéalement d'établir des relations commerciales pérennes avec les entreprises classiques, une part de l'activité correspond à des commandes ponctuelles qui émanent de clients épisodiques et qui servent de variable d'ajustement face aux aléas de la demande.

La prospection commerciale ne s'avère pas des plus simples. Ainsi, l'EI se sent soumise aux mêmes contraintes que n'importe quelle entreprise en termes de concurrence. Sa dimension sociale ne constitue un argument de vente qu'auprès des entreprises classiques qui la sollicitent dans le cadre d'une politique d'achat responsable. De plus, même dans ce cas, il convient d'offrir une prestation satisfaisante seule susceptible de fidéliser les clients : " $O n$ essaie vraiment de se présenter comme un prestataire lambda qui a des préoccupations de délais, de qualité de la prestation, au même titre que n'importe quel prestataire. Et qui a comme préoccupation, on va dire première, au vu du client, sa satisfaction. C'est-à-dire que toute la partie insertion, à la limite, le client il n'est pas censé ni en prendre connaissance ni en avoir tellement

7. Ces deux dilemmes sont dans les faits imbriqués, mais nous les présentons séparément afin de clarifier les difficultés que pose la primauté accordée au principe civique dans les SIAE. 
conscience au vu de la prestation. " (Directrice commerciale, EI, grande école de commerce, deux ans d'expérience dans la fonction)

Face à la nécessité de vendre ses prestations pour survivre, l'EI se trouve confrontée au dilemme suivant. Dans quelle mesure faut-il accepter certains contrats financièrement intéressants, même s'ils ne sont pas porteurs en termes d'insertion ? Symétriquement, faut-il accepter certains contrats porteurs en termes d'insertion, même s'ils ne sont pas financièrement intéressants ? Le principe civique qui guide l'EI se trouve ainsi en tension avec le principe marchand : "Est-ce qu'on choisit de prendre ce chantier là tout en sachant que ben... Parfois, ça va être un chantier super pour nos employés, mais très peu rentable. Est-ce qu'on prend quand même, sachant qu'on a une probabilité de perdre des ronds dans l'histoire? Ou alors, un chantier qui va être super dur, qui est assez loin, etc. Est-ce qu'on y va quand même, tout en sachant qu'au niveau social, ça va franchement ne pas être terrible? (...) Et donc, il y a des moments où il faut trancher. " (Directrice de l'antenne $\mathrm{n}^{\circ} 1$, EI, grande école de commerce, cinq ans d'expérience dans la fonction)

L'activité de l'ETTI provient, pour partie, de l'achat responsable, qui représente un tiers de son chiffre d'affaires. Mais ce dernier résulte désormais, à hauteur des deux tiers, des clauses sociales dans les marchés publics. Ce dispositif oblige les entreprises attributaires à faire exécuter, par des personnes en insertion, un pourcentage des heures de main-d'œuvre nécessaires à l'exécution du marché public. Elles sont particulièrement répandues dans le secteur du BTP, sous l'effet du programme national de rénovation urbaine, lancé en 2003.

Même s'il existe plusieurs modalités pour les honorer, les entreprises classiques choisissent en priorité de faire appel à du personnel mis à disposition. L'obligation, pour les entreprises attributaires, d'avoir recours à du public en insertion facilite alors grandement la prospection commerciale de l'ETTI. Pour autant, rien ne garantit que ce volume d'activité lui revienne. Elle entre en effet en concurrence avec diverses structures proposant aussi de la mise à disposition, comme les autres ETTI, les AI, les groupements d'employeurs pour l'insertion et la qualification, voire les agences d'intérim classiques. Pour tirer parti du dispositif des clauses sociales, l'ETTI doit maîtriser ses tarifs afin d'attirer les entreprises utilisatrices : "J'ai envie de dire qu'encore, dans la clause d'insertion, on est dans le moinsdisant prix souvent, parce que "bon je suis obligé de faire de la clause, mais il ne faut pas que ça me coûte». (...) Parfois, ils vont consulter deux ou trois SIAE en disant "Ah ben non, je prends lui, il est moins cher". "Très bien. Nous on apporte ça, ça et ça. Dites-nous ce qui peut faire la différence". On est sur de la mise en concurrence » (responsable antenne n², ETTI, maîtrise de sciences économiques, douze ans d'expérience dans la fonction).

La plupart des entreprises classiques envisagent donc le dispositif des clauses sociales uniquement comme un coût qu'il faudrait réduire et s'y adaptent a minima (Semenowicz, 2017). Afin d'éviter le paiement de pénalités, la conformité aux obligations contractuelles est parfois obtenue par un recours massif à du personnel en insertion sur la fin du chantier, dans l'urgence. De plus, les entreprises utilisatrices font généralement appel à l'ETTI sur des missions courtes correspondant aux postes les moins qualifiés (manœuvre...) Cette 
appréhension des clauses sociales n'est bien évidemment pas propice au développement des compétences des personnes en insertion : «Les deux premiers mois, ils négligent l'affaire tout à fait. Et quand ils entendent parler d'une amende à payer à la fin du chantier sills ne respectent pas, là c'est direct deux/trois personnes pour le lendemain pendant deux mois pour faire les heures (...) A vrai dire, est-ce quill y a des sociétés qui soubaitent apprendre quelque chose à ces gens-là? Il y en a peu. La plupart les exploitent, je le dis concrètement car je le vois sur les chantiers. Tout ce qui est métiers à risques, ils les mettent dessus. Malheureusement, ça c'est $50 \%$ des clients. " (Chargé du développement commercial, ETTI, BTS - brevet de technicien supérieur - Action commerciale, deux ans d'expérience dans la fonction)

Articuler le principe civique avec le principe marchand dans un compromis s'avère donc complexe. Il en va de même avec le principe industriel.

\subsection{Sélectionner à l'entrée ?}

Si l'on s'en tient au slogan de l'IAE selon lequel "nul n'est inemployable ", une SIAE est censée pouvoir employer tout type de public rencontrant des difficultés sociales ou professionnelles particulières. Une tension découle pourtant du possible décalage entre les caractéristiques des personnes en insertion et les attentes des entreprises clientes.

Ainsi, au quotidien, la question la plus pressante pour l'EI est d'assurer une prestation correcte avec du personnel supposé peu productif. L'objectif affiché de satisfaction des entreprises clientes soulève en effet la question de sa compatibilité avec le profil des salariés en insertion. Leur niveau de productivité peut avoir des répercussions en termes de délai, de qualité, etc., qui font que les contraintes de la production s'avèrent difficiles à articuler avec la mission sociale. En outre, l'accompagnement social des personnes en insertion nécessite fréquemment qu'elles soient absentes pour accomplir diverses démarches. Par conséquent, une sélection parmi les publics agréés s'opère lors du recrutement, en fonction de leur degré d'éloignement de l'emploi. L'EI se défend d'effectuer un écrémage systématique, mais elle reconnaît gérer le problème par la recherche d'une hétérogénéité au sein des publics accueillis, de façon à parvenir à un certain équilibre : «Le chargé d'insertion entend les demandes des chefs d'équipe. Si le chef d'équipe dit "Écoute, là, moi concrètement, en ce moment, j'ai plutôt une équipe où les personnes sont très lentes, elles ont vachement de mal à comprendre, franchement je ne vais pas pouvoir tenir mes objectifs de production, y'a rien à faire à ça, je ne vais pas y arriver", le chargé d'insertion, le recrutement suivant, il va essayer de faire attention à prendre une personne avec un profil un peu différent par rapport à ça. Et à équilibrer pour se retrouver quand même avec des profils très hétérogènes pour quion n'ait pas ce genre de problématique là. » (Responsable accompagnement social, EI, diplôme d'État d'assistante sociale, dix ans d'expérience dans la fonction)

Ces éléments pourraient laisser penser que le principe civique n'est pas compatible avec le principe industriel. Pourtant, la position revendiquée au sein de l'EI est qu'ils ne seraient pas forcément contradictoires. Au contraire, c'est lorsque l'on parviendrait à les concilier 
que la mission sociale pourrait pleinement être remplie. En devant satisfaire les exigences des clients que représentent les entreprises classiques, les personnes en insertion seraient placées dans des conditions de production très formatrices car proches de celles rencontrées sur le marché du travail de droit commun : «Si on fait une prestation de grande qualité, c'est hyper valorisant pour les employés en insertion, le contraire étant dévalorisant. Donc, il y a quand même un côté vertueux où le côté économique et le côté social se rejoignent. Et d'un autre côté, au niveau pédagogique, plus on est cadrants sur les retards, les absences, le comportement au travail, plus c'est bénéfique pour les salariés en insertion qui sont en quelque sorte en apprentissage ou en réapprentissage du monde du travail et de la vie professionnelle. " (Directrice de l'antenne $\mathrm{n}^{\circ} 1, \mathrm{EI}$, grande école de commerce, cinq ans d'expérience dans la fonction)

L'ETTI opère également une sélection à l'entrée des publics sur la base d'un entretien préalable. Il vise à repérer, pour chaque personne, les compétences détenues et l'ampleur des problématiques sociales. L'ETTI justifie cette entorse à la devise de l'IAE par une répartition des rôles entre les différentes SIAE.

Il existe en effet implicitement, au sein de l'IAE, un parcours d'insertion formalisé. L'ACI en constitue la première étape, l'AI ou l'EI l'étape intermédiaire et l'ETTI l'étape finale (Gérôme, 2017). Si la personne s'avère trop éloignée de l'emploi pour différentes raisons, l'ETTI la dirige donc vers un autre type de structure, plus adaptée à sa situation : "Il faut qu'elle soit éloignée de l'emploi, mais pas trop non plus (...) Ça nécessite quand même une certaine autonomie. Il faut que la personne puisse se déplacer sur un chantier, changer de chantier, faire face à différents interlocuteurs, un chef d'équipe un jour, un conducteur de travaux un autre, etc. Il faut une personne qui soit un peu prête à l'emploi quand même. Parce que sinon, le parcours avec nous, il ne va pas être pertinent. " (Chargée d'optimisation parcours, ETTI, master en droit social, trois ans d'expérience dans la fonction)

Les spécificités du public en insertion peuvent en effet générer des insatisfactions du côté des entreprises clientes (absentéisme, difficultés relationnelles...). À défaut d'encadrant technique, l'ETTI met en place un suivi régulier, effectué par des chargés de mission, sur le lieu d'exercice pour prévenir ces situations. Leur présence sur les chantiers permet ainsi d'échanger avec les chefs de travaux. Il s'agit de s'assurer de la qualité de la relation qui s'établit entre la personne en insertion et son supérieur hiérarchique dans l'entreprise utilisatrice. Cette relation est en effet présentée comme essentielle dans la réussite d'une mission. Contrairement aux agences d'intérim classiques, la mise à disposition ne se limite donc pas à la simple délégation de l'intérimaire. L'attention accordée par l'ETTI au suivi, dans la mission, contribue à dénouer les éventuelles tensions. Il s'agit de satisfaire les entreprises clientes, malgré la spécificité du public mis à disposition : "Une fois que la mission démarre, nous on essaye de repasser sur le chantier sous dix jours pour faire un point avec le chef. Sinon, on l'appelle quand même en fin de semaine pour savoir comment ça s'est passé. L'intérimaire on l'appelle avant, c'est-à-dire sous deux jours. On l'appelle pour lui dire "Comment ça va ?", "Est-ce que vous vous sentez bien ?", "Est-ce que vous êtes encadré ?". Histoire de voir... En fait, le but de tout cela, c'est de prévenir. Prévenir au maximum. Etre au 
courant de ce qui se passe pour que si à un moment, il y a un petit malaise, on puisse rapidement éteindre le début d'incendie " (responsable antenne $\mathrm{n}^{\circ} 3$, ETTI, master politique de la ville, deux ans d'expérience dans la fonction).

Lorsque le suivi fait apparaître une insatisfaction de l'entreprise utilisatrice, la réaction de l'ETTI est double. D'une part, elle mène un travail pédagogique auprès de l'intérimaire pour que celui-ci révise, le cas échéant, son comportement. D'autre part, elle s'efforce de résister aux demandes de changement d'intérimaire lorsqu'elles surviennent. Pour maintenir la personne en insertion dans sa mission, l'ETTI change alors de registre en mobilisant le principe civique. Elle peut, par exemple, mettre en avant les difficultés de l'intérimaire pour justifier certains de ses écarts auprès de l'entreprise utilisatrice. Par ailleurs, lorsque la mise à disposition s'inscrit dans le cadre d'une clause sociale, un autre moyen est de rappeler à l'entreprise son obligation contractuelle. Les opérationnels sur le chantier ont en effet tendance à traiter avec l'ETTI comme s'il s'agissait d'une agence d'intérim classique, en oubliant le cadre dans lequel se déroule la mise à disposition. Or, la clause sociale implique nécessairement de déléguer du public en insertion, et en rappelant cette exigence, l'ETTI peut alors relativiser le déficit de productivité reproché par l'entreprise utilisatrice. Pour autant, l'ETTI n'a pas toute latitude en la matière. En effet, lorsque l'entreprise utilisatrice l'impose, elle est contrainte de procéder à un changement d'intérimaire malgré tout.

Comme le principe marchand, le principe industriel s'avère complexe à articuler avec le principe civique. Ces difficultés peuvent être minorées dans le cadre de partenariats établis dans la durée avec les entreprises classiques.

\section{3. Établir des partenariats pérennes avec les entreprises classiques}

L'achat responsable et le dispositif des clauses sociales dans les marchés publics constituent les principaux motifs de recours aux SIAE pour les entreprises classiques. Comme nous l'avons vu, tous deux aboutissent généralement à des transactions s'inscrivant dans les mondes marchand et industriel, dont l'articulation avec le principe civique s'avère délicate.

Toutefois, ces transactions peuvent parfois déboucher sur de véritables partenariats au service de l'insertion, à la condition d'un engagement fort des entreprises classiques. Quelques grands groupes font en effet preuve d'un "volontarisme social " par leur action résolue en matière d'insertion des publics éloignés de l'emploi (Cusin \& Charreire-Petit, 2015) ${ }^{8}$. Leurs motivations mêlent des fins sociétales, de communication et de résolution de problématiques de gestion des ressources humaines (turnover ou pénurie de maind'œuvre sur certaines fonctions). La lutte contre l'exclusion professionnelle accède ainsi au rang de "projet» (Boltanski \& Chiapello, 1999) légitime en leur sein. Afin de le

8. Les partenariats que nous avons observés dans le cadre de nos monographies concernent exclusivement des grands groupes, mais certaines SIAE en ont également noué avec des petites et moyennes entreprises. 
mener à bien, ces grands groupes s'appuient, conformément à leur organisation de type "firme-réseau ", sur des partenaires spécialisés dans l'insertion, en l'occurrence, les SIAE. S’inscrivant dans la durée, les collaborations qui en découlent ambitionnent d'articuler le principe connexionniste avec le principe civique.

Ainsi, pour certains grands groupes, l'EI assure des opérations de sous-traitance qui se singularisent par leur ancienneté et peuvent être considérées comme de véritables partenariats. Leur pérennité s'explique par un engagement délibéré de ces grands groupes. Les contrats sont en effet conclus pour des périodes pluriannuelles et reconduits tacitement à terme. L'EI s'efforce de nouer de tels partenariats en repérant des activités peu qualifiées susceptibles d'être externalisées par ces entreprises.

Cette stratégie explique que l'EI se caractérise par la multi-activité plutôt que par la spécialisation. Elle se distingue ainsi par sa capacité à développer en permanence de nouvelles activités pour lesquelles une demande de ces grands groupes existe. La variété des activités assurées est impressionnante, puisqu'elles peuvent concerner le bâtiment, la logistique, le nettoyage écologique de véhicules, le débarras, le tri des déchets... leur point commun étant de correspondre à des tâches peu ou pas qualifiées.

La compétence dont peut se prévaloir l'EI dans un monde connexionniste réside alors dans le management d'équipes de production composées de personnes éloignées de l'emploi : "Ce que je dis souvent aux équipes c'est, moi je pense que l'insertion, on doit pouvoir se développer sur des niches de métiers qui n'existent pas. Et c'est quasiment à l'insertion d'innover sur de nouveaux métiers pour pouvoir créer de nouveaux marchés, rentrer en contact avec les entreprises. " (Directeur de l'antenne $\mathrm{n}^{\circ} 2, \mathrm{EI}$, grande école de commerce, un an d'expérience dans la fonction)

L'EI s'efforce de promouvoir, auprès des entreprises classiques, une conception exigeante de l'achat responsable se traduisant par un engagement pérenne. Par rapport aux commandes ponctuelles caractéristiques du monde marchand, pour l'EI, de tels partenariats constituent une opportunité en offrant une certaine stabilité. Les contraintes habituelles de la sous-traitance en sont atténuées et les activités de production peuvent davantage être organisées à partir des besoins des personnes en insertion. La conciliation des principes civique et connexionniste passe alors par une réflexion sur l'organisation du travail : " Il faut bien connaître son activité, voir comment on peut l'adapter au mieux pour accueillir un certain public et voir comment on intègre des gens. De toute façon, sur une activité, il faut qu'il $y$ ait un noyau dur, il faut qu'il y ait des gens qui sachent faire, et après, on peut se permettre d'intégrer des gens qu'on va pouvoir accompagner plus longtemps. C'est ce qu'on arrive à faire, mais la chance c'est qu'on est sur une activité pérenne et stable. Donc, ça permet de se dédouaner de toute la problématique "demain qu'est-ce que je donne comme boulot ?" Là, on ne se pose pas la question de voir si demain, il y a du boulot. Il y a du boulot, donc c'est plutôt comment on va le faire, comment on va l'organiser et là, on fait un vrai travail d'insertion, là ça devient vraiment bien. " (Directeur de l'antenne ${ }^{\circ} 3$, EI, DEA en économie internationale, quinze ans d'expérience dans la fonction) 
De même, l'ETTI a fidélisé un certain nombre d'entreprises utilisatrices qui font systématiquement appel à elle pour honorer leurs obligations dans le cadre des clauses sociales. De tels partenariats génèrent tout d'abord des ressources régulières, contrairement aux commandes ponctuelles qui lui sont habituellement adressées. Ils constituent également un cadre propice pour amener ces entreprises à aborder les clauses sociales de manière qualitative, afin de favoriser l'insertion durable des bénéficiaires à l'issue du dispositif (Havet $\&$ al., 2016). Les recommandations de l'ETTI concernent tout d'abord la répartition des heures d'insertion sur les différents métiers concernés : "Une entreprise a 1500 heures à faire. Comment va-t-elle les faire? Soit elle va dire "Voilà, j’ai 1500 heures à faire, j’ai découpé le truc." Alors, il y en a certaines qui sont très méthodiques. "Moi je vais prendre un tiers sur le gros ouvre, là je vais prendre l'électricien, il a tant de milliers d'euros de chantier, donc, ça va faire tant d'heures, le plombier pareil, lui, l'ascensoriste, il fera cinquante heures, l'espaces verts, il en fera cent"s". Je dis "Attendez, on va arrêter. Il faut du sens. Cinquante heures chez un ascensoriste, il n'y a pas d'ascensoriste en insertion. Reportez les heures sur autre chose. Redessinons ensemble la cible et l'exécution de vos heures"." "(Responsable antenne n², ETTI, maîtrise de sciences économiques, douze ans d'expérience dans la fonction).

L'intervention de l'ETTI auprès des entreprises utilisatrices vise ensuite la construction de parcours d'insertion pour les bénéficiaires des clauses sociales. Une négociation s'instaure pour obtenir des missions longues qui permettent à l'ETTI, si la mise à disposition se déroule bien, une diversification des activités de l'intérimaire. Un rôle clé de l'ETTI consiste donc à associer l'entreprise utilisatrice au parcours d'insertion du bénéficiaire, afin de lui faire acquérir de nouvelles compétences : "On essaie vraiment de travailler sur des longs parcours, de travailler sur toute la durée du chantier. Même sill y a des difficultés, essayer de trouver des solutions (...) Il y a aussi les responsables ressources humaines qui intègrent l'insertion dans leurs recrutements et dans leur façon de voir les choses. Donc, ça veut dire qu'on ne fait plus des contrats d'une semaine que l'on renouvelle. " (Responsable antenne $\mathrm{n}^{\circ} 1$, ETTI, DUT Techniques de commercialisation, trois ans d'expérience dans la fonction)

Afin d'atteindre un compromis civique-connexionniste, les deux SIAE étudiées cherchent donc à établir, avec les entreprises classiques, des partenariats dans la durée. Il s'agit ainsi de contenir partiellement les contraintes issues tant du monde marchand que du monde industriel.

\section{Conclusion}

La double dimension économique et sociale des SIAE ne peut être assumée que par des compromis entre différents principes supérieurs communs. Le compromis civique-marchand répond à l'impératif de décrocher des commandes, desquelles provient l'essentiel du financement et qui permettent une mise en situation de travail des personnes en insertion. Toutefois, le caractère ponctuel des contrats obtenus et la nature des activités assurées ne favorisent pas toujours le développement des compétences des publics. Le compromis civique-industriel place les personnes en insertion dans des conditions de production for- 
matives par leur proximité avec des emplois de droit commun. À l'inverse, il peut conduire à un écrémage plus ou moins marqué des publics afin de répondre aux contraintes de la production.

Face à ces dilemmes, les deux SIAE étudiées se rejoignent par leur tentative commune d'élaborer un compromis civique-connexionniste via des partenariats pérennes avec les entreprises classiques. D'une part, ce type de relation améliore la viabilité financière à moyen terme, tout en créant des conditions plus favorables au développement des compétences des personnes en insertion. D’autre part, la relative stabilité des activités ainsi obtenue autorise la conception d'une organisation du travail plus inclusive qui amoindrit la sélection des publics à l'entrée. Les SIAE se trouvent ainsi en meilleure position pour assumer leur rôle de "sas vers l'emploi ", qu'elles conçoivent comme la transmission de savoir-être davantage que de savoir-faire.

Analysant la conciliation entre mission sociale et contraintes économiques sur la base d'une enquête quantitative réalisée à l'échelle nationale, Rémy (2017) constate une diversité des stratégies adoptées par les SIAE. Les arbitrages ne se limitent pas à privilégier l'une ou l'autre des dimensions, mais s'étendent à une palette de solutions. L'auteure relève en particulier que "les structures entretenant des liens privilégiés avec des entreprises (hors IAE) estiment plus souvent être en mesure de définir et de valider le projet professionnel de leurs salariés, d'améliorer leur capacité d'exécution des tâches et leurs autres capacités (dynamisme, esprit d'équipe, initiative, motivation, maîtrise de soi), mais aussi de prendre en charge leurs addictions, de résoudre leurs problèmes d'accès aux droits ainsi que d'améliorer leur estime de soi» (2017, p. 26). Le compromis civique-connexionniste que nous avons identifié dans nos monographies permet d'éclairer cette corrélation. À défaut de résoudre totalement les dilemmes auxquels sont confrontées les SIAE, il permet toutefois de les modérer. La généralisation de partenariats établis dans la durée avec les entreprises classiques ne peut toutefois survenir qu'à condition que ces dernières s'engagent résolument en faveur de l'insertion des publics éloignés de l'emploi, en développant des politiques d'achat responsable exigeantes ou en participant activement aux clauses sociales.

\section{Bibliographie}

Autès M. (1999), Les Paradoxes du travail social, Paris, Dunod.

Baron C., Bureau M.-C., Le Dantec E., Nivolle P. (1994), Les intermédiaires de l'insertion, Paris, CEE.

Baudry B. (1995), L'économie des relations interentreprises, Paris, La Découverte.

Bellit S. (2017), «L'insertion par l'activité économique en 2016 », DARES Résultats, nº 74.

Boltanski L., Chiapello E. (1999), Le Nouvel esprit du capitalisme, Paris, Gallimard.

Boltanski L., Thévenot L. (1991), De la justification, Paris, Gallimard. 
Bucolo E., Eme B., Gardin L. (2012), «Les entreprises sociales et l'insertion par l'activité économique en France », in Gardin L., Laville J.-L., Nyssens M. (dir.), Entreprise sociale et insertion, Paris, Desclée De Brouwer, pp. 185-216.

Castra D. (2003), Linsertion professionnelle des publics précaires, Paris, Presses Universitaires de France.

Cusin J., Charreire-Petit S. (2015), "Vers l'identification des leviers, des risques et des arbitrages dans le déploiement d'une pratique RSE tournée vers les publics éloignés de l'emploi ", Revue de l'organisation responsable, vol. 10, n² 2, pp. 58-74.

Defalvard H., Cervera M. (2009), «Accompagnement dans et vers l'emploi : profits et pertes dans les structures d'insertion par l'activité économique ", Travail et Emploi, $\mathrm{n}^{\circ} 119$, pp. 51-62.

Ebersold S. (2001), La naissance de l'inemployable ou l'insertion aux risques de l'exclusion, Rennes, Presses universitaires de Rennes.

Eme B. (1994), "Insertion et économie solidaire », in Eme B., Laville J.-L. (dir.), Cohésion sociale et emploi, Paris, Desclée de Brouwer, pp. 157-194.

Eymard-Duvernay F. (2004), Économie politique de l'entreprise, Paris, La Découverte.

Gérôme C. (2017), Les experts de l'insertion. Sociologie des fédérations associatives de l'insertion par l'activité économique, thèse de doctorat en sociologie, université Paris-Est.

Havet N., Penot A., Plantier M. (2016), « Le devenir des bénéficiaires des clauses sociales des marchés publics ", Formation Emploi, n 136, pp. 121-143.

Mauger G. (2001), « Les politiques d'insertion : une contribution paradoxale à la déstabilisation du marché du travail ", Actes de la recherche en sciences sociales, n 136-137, pp. 5-14.

Rémy V. (2016), "Les structures de l'insertion par l'activité économique : un accompagnement très diversifié durant le parcours d'insertion ", DARES Analyses, n 19.

Rémy V. (2017), «Comment les structures de l'insertion par l'activité économique concilient-elles leur mission d'accompagnement et leur activité ? ", Document d'études de la DARES, $\mathrm{n}^{\circ} 205$.

Semenowicz P. (2017), Collaborer pour insérer? Les partenariats sociaux dans l'insertion par l'activité économique, Rennes, Presses universitaires de Rennes. 